\title{
Nicotinamide Administration Improves Remyelination after Stroke
}

\author{
Congxiao Wang, Yi Zhang, Jie Ding, Zhen Zhao, Cheng Qian, Ying Luan, and Gao-Jun Teng \\ Jiangsu Key Laboratory of Molecular and Functional Imaging, Department of Radiology, Zhongda Hospital, Medical School of \\ Southeast University, Nanjing 210009, China
}

Correspondence should be addressed to Gao-Jun Teng; gjteng@vip.sina.com

Received 15 December 2016; Revised 16 February 2017; Accepted 13 March 2017; Published 1 June 2017

Academic Editor: Long-Jun Wu

Copyright ( 2017 Congxiao Wang et al. This is an open access article distributed under the Creative Commons Attribution License, which permits unrestricted use, distribution, and reproduction in any medium, provided the original work is properly cited.

\begin{abstract}
Aims. Stroke is a leading cause of morbidity and mortality. This study aimed to determine whether nicotinamide administration could improve remyelination after stroke and reveal the underlying mechanism. Methods. Adult male C57BL/6J mice were intraperitoneally (i.p.) administered with nicotinamide $(200 \mathrm{mg} / \mathrm{kg}$, daily) or saline after stroke induced by photothrombotic occlusion of the middle cerebral artery. FK866 (3 mg/kg, daily, bis in die), an inhibitor of NAMPT, and ANA-12 (0.5 mg/kg, daily), an antagonist of tropomyosin-related kinase B (TrkB), were administered intraperitoneally $1 \mathrm{~h}$ before nicotinamide administration. Functional recovery, MRI, and histological assessment were performed after stroke at different time points. Results. The nicotinamide-treated mice showed significantly lower infarct area $7 \mathrm{~d}$ after stroke induction and significantly higher fractional anisotropy (FA) in the ipsilesional internal capsule (IC) $14 \mathrm{~d}$ after stroke induction than the other groups. Higher levels of $\mathrm{NAD}^{+}, \mathrm{BDNF}$, and remyelination markers were observed in the nicotinamide-treated group. FK866 administration reduced $\mathrm{NAD}^{+}$and BDNF levels in the nicotinamide-treated group. ANA-12 administration impaired the recovery from stroke with no effect on $\mathrm{NAD}^{+}$and BDNF levels. Furthermore, lesser functional deficits were observed in the nicotinamidetreated group than in the control group. Conclusions. Nicotinamide administration improves remyelination after stroke via the $\mathrm{NAD}^{+} / \mathrm{BDNF} /$ TrkB pathway.
\end{abstract}

\section{Introduction}

Ischemic stroke is a leading cause of mortality and morbidity [1]. To date, recombinant tissue plasminogen activator (rtPA) is the only approved drug for the treatment of acute ischemic stroke with a $4.5 \mathrm{~h}$ window for therapeutic intervention after ischemic stroke onset [2-6]. However, very few patients with acute ischemic stroke receive this therapy. Moreover, as many as half of the patients with ischemic stroke receiving rtPA treatment survive and suffer longterm disability. Therefore, there is considerable interest in developing new treatment modalities for stroke, with focus on restorative therapeutics [7].

Over the past decades, the majority of studies have focused on the insults suffered by the gray matter after stroke, while less attention has been paid to white matter injuries [8]. However, white matter constitutes about $50 \%$ of the brain volume in humans, and clinical data suggest that ischemic stroke in most cases damages both white matter and gray matter [9]. The white matter, constituted of axons and glial cells including oligodendrocytes, astrocytes, and microglia, is vulnerable to ischemic insults and can be considerably damaged also by brief focal ischemic episodes. Notably, white matter injury is the major cause of functional disability after ischemic stroke [9-11]. In the central nervous system, information is rapidly transmitted between different brain regions along axons, which are enveloped in a multilayered membrane sheath formed by oligodendrocytes that increase the conduction speed of action potentials via accelerating impulse conduction $[12,13]$. Myelin sheath has a vital role for cell interaction involving information exchange between oligodendrocytes and axons; it provides nutrition support for neurons and is important for the motor, sensory, and cognitive functions of the nervous system $[13,14]$. Damage to oligodendrocytes caused by ischemia causes loss of action potential propagation through myelinated axons also when the neuronal integrity is preserved [15]. Therefore, we believe that it is important to develop an effective therapy to promote 
remyelination after stroke in consideration of the important role of myelin in the functioning of the nervous system and the consequences of its damage after stroke insult.

Nicotinamide (NAM) is the amide derivative of nicotinic acid (also known as vitamin B3 or niacin). NAM, a precursor of nicotinamide adenine dinucleotide $\left(\mathrm{NAD}^{+}\right)$, is converted into $\mathrm{NAD}^{+}$by nicotinamide phosphoribosyltransferase (NAMPT), a rate-limiting enzyme in $\mathrm{NAD}^{+}$biosynthesis in vivo [16]. Despite structural similarities, NAM has different functions and clinical uses from nicotinic acid. NAM is an approved drug for medical use and shows a good safety profile at high doses. Moreover, the vasodilatory side effects of nicotinic acid such as hypotension, headache, and flushing are not observed with NAM [17]. In the recent years, NAM has been used in animal models and clinical trials to assess its efficacy in treating a variety of diseases including type 1 diabetes, acute lung injury, Friedreich's ataxia, skin cancer chemoprevention, Parkinson's disease (PD), Huntington's disease (HD), and ischemic stroke [18-22]. To date, the majority of the studies have investigated the therapeutic effects of NAM on the acute phase of ischemic stroke, and different mechanisms involved in its protective effect have been described [19] yet overlook the possible effect of longterm NAM administration on remyelination after stroke. Our study aims to investigate whether long-term NAM administration could be used as a restorative therapy to promote the remyelination after stroke and to reveal the underlying mechanism.

\section{Methods}

2.1. Photothrombotic Middle Cerebral Artery Ischemic Stroke Model. All animal experiments in this study were approved by the Institutional Animal Care and Use Committee (IACUC) of Southeast University (approval ID: SYXK-2010.4987). Adult male C56BL/6J mice (20.025.0 g, 8-10 wk, Comparative Medicine Centre of Yangzhou University [SCXK (SU) 2012-0004], Yangzhou, China) were used in this study. Photothrombotic ischemic stroke was induced by a well-established previously reported procedure $[23,24]$. Briefly, mice were anesthetized with pentobarbital (50 mg/kg; 1\% in sterile saline), and body temperature was maintained at $\sim 37^{\circ} \mathrm{C}$ with a heating pad. An incision was made between the right orbit and the external auditory canal to separate the temporalis muscle from the dura mater, and the proximal section of the middle cerebral artery was exposed. The photosensitizer Rose Bengal $(0.025 \mathrm{mmol} / \mathrm{kg}$, Sigma-Aldrich, Shanghai, China) was injected intravenously, and after $2 \mathrm{~min}$, a laser beam (diameter: $0.1 \mathrm{~mm}$, wavelength: $532 \mathrm{~nm}$, Shanghai Laser \& Optics Century Co. Ltd., Shanghai, China) was focused on it. The skin was then sutured and mice were returned to the cages to regain consciousness.

2.2. Drug Administration and Experimental Groups. Each experimental group included 16 mice. Four experimental groups were identified as follows: Group 1, mice were treated with NAM alone; Group 2, mice were treated with NAM and ANA-12; Group 3, mice were treated with NAM and FK866; and Group 4, mice were treated with saline (control group). NAM (200 mg/kg, Sigma-Aldrich, Shanghai, China) was injected intraperitoneally (i.p.) daily for $14 \mathrm{~d}$; the first injection occurred $1 \mathrm{~h}$ after stroke induction. ANA-12 $(0.5 \mathrm{mg} / \mathrm{kg}$, Sigma-Aldrich, Shanghai, China), a selective antagonist of tropomyosin receptor kinase B (TrkB), was injected intraperitoneally $1 \mathrm{~h}$ before NAM injection. FK866 (3 mg/kg, Selleck Chemicals, Shanghai, China), an antagonist of nicotinamide phosphoribosyltransferase (NAMPT), was intraperitoneally injected twice a day for $14 \mathrm{~d}$, the first dose administered $1 \mathrm{~h}$ before NAM injection. The same amount of volume of saline solution was intraperitoneally administered to the control group.

2.3. Magnetic Resonance Imaging. Magnetic resonance imaging (MRI) was performed using a $7 \mathrm{~T}$ small animal magnetic resonance system (PharmaScan; Bruker, Ettlingen, Germany). Mice were anesthetized with 1\% isoflurane (Shandong Keyuan Pharmaceutical Co. Ltd., Shandong, China), heart rate maintained at $\sim 100 \mathrm{bpm}$. At $24 \mathrm{~h}$ and $7 \mathrm{~d}$ poststroke, turbo spinecho sequence T2-weighted images (T2WI) were collected with the following set of parameters: field of view $=2 \mathrm{~cm} \times$ $2 \mathrm{~cm}$, slice thickness $=1 \mathrm{~mm}$, slices $=15$, interslice distance $=1 \mathrm{~mm}$, repetition time $=3 \mathrm{~s}$, averages $=1$, matrix size $=$ $256 \times 256$, flip angle $=180^{\circ}$, and total scan time for image acquisition: $1 \mathrm{~min} 20 \mathrm{~s}$. For diffusion tensor imaging (DTI), an echo-planar imaging (EPI) sequence was used to acquire 30 distinct diffusion directions. Five reference images were obtained at $14 \mathrm{~d}$ with the following set of parameters: field of view $=2 \mathrm{~cm}$, slice thickness $=0.6 \mathrm{~mm}$, slices $=20$, matrix size $=128 \times 128$, repetition time $=5 \mathrm{~s}$, average $=2$, and total scan time for DTI: 23 min 20s. The infarct volume was calculated with ImageJ software (National Institutes of Health, Bethesda, MD) using the equation: infarct sizes $\%=\sum\{$ infarct area - (ipsilateral hemisphere - contralateral hemisphere) $\} \times$ $100 / \sum$ (contralateral hemisphere). Fractional anisotropy (FA) was measured from the tensor map in the ipsilateral internal capsule (IC) with ParaVision 5.0 software (Bruker, Ettlingen, Germany). Fiber tracking was carried out using TrackVis (Version 0.5.2.1, Center for Biomedical Imaging, Department of Radiology, Massachusetts General Hospital) software and Diffusion Toolkit (version 0.6.2.1) in the ipsilateral internal capsule (IC) of the brain.

2.4. Immunohistochemistry. Mice brains were extracted at $7 \mathrm{~d}$ and $14 \mathrm{~d}$ and fixed in $4 \%$ paraformaldehyde overnight at $4^{\circ} \mathrm{C}$. They were subsequently immersed in $30 \%$ sucrosecontaining tubes until they sunk to the bottom, before being transferred onto a freezing microtome (CM1950, Leica Biosystems Nussloch GmbH, Nussloch, Germany) to obtain slices of $10 \mu \mathrm{m}$ thickness. To confirm the expression of $\mathrm{O} 4$, brain-derived neurotrophic factor (BNDF), and myelin basic protein (MBP), immunofluorescence staining was carried out. Brain sections were rehydrated using phosphate-buffered saline (PBS) for $10 \mathrm{~min}$ before being blocked with PBT buffer (3\% BSA and $0.1 \%$ Triton X-100 in PBS) for $1 \mathrm{~h}$ at $25^{\circ} \mathrm{C}$. Further, brain sections were incubated with rabbit anti-mouse BDNF polyclonal antibody (1:500 dilution; Abcam, Cambridge, UK), rabbit anti-mouse MBP 
antibody (1:500 dilution; Abcam, Cambridge, UK), and mouse anti-mouse monoclonal antibody (1:100 dilution; Merck Millipore, Shanghai, China) separately overnight at $4^{\circ} \mathrm{C}$. After washing three times with PBS, sections were incubated with the secondary antibodies rabbit anti-mouse Alexa Fluor 488 (1:500 dilution; Invitrogen, part of Thermo Fisher Scientific, Carlsbad, CA) for O4 staining, goat anti-rabbit Alexa Fluor 546 (1:500 dilution; Invitrogen, part of Thermo Fisher Scientific, Carlsbad, CA) for BDNF and MBP staining, for $2 \mathrm{~h}$ at $25^{\circ} \mathrm{C}$. Finally, after washing for three times with PBS, nuclear DNA was stained with DAPI (SouthernBiotech, Birmingham, AL). Brain sections were observed using a ZEISS microscope and image analysis was carried out using the Image-Pro Plus software (Media Cybernetics, Rockville, $\mathrm{MD})$. All staining was done identically and at the same time on the groups being compared.

2.5. Western Blot Analysis. Brain samples were homogenized in RIPA buffer (KeyGEN BioTECH, Nanjing, China) containing $1 \%$ protease inhibitor cocktail (Roche Applied Sciences, Penzberg, Germany), and centrifuged at 15,000 rpm for $15 \mathrm{~min}$ at $4^{\circ} \mathrm{C}$ (Centrifuge 5417R; Eppendorf, Hamburg, Germany). Supernatants were transferred into a labeled tube. After quantification using the BCA protein quantification kit (KeyGEN BioTECH, Nanjing, China), proteins were denatured with a loading buffer at $100^{\circ} \mathrm{C}$. Equal amounts of proteins were separated using SDS-PAGE and transferred to a PVDF membrane (Bio-Rad Laboratories, Shanghai, China). The PVDF membrane was blocked with 5\% milk at $25^{\circ} \mathrm{C}$ for $1 \mathrm{~h}$ before incubation with primary antibody for BDNF (1:500 dilution; Abcam, Cambridge, UK), MBP (1:1000 dilution, Abcam, Cambridge, UK) and $\beta$-actin ( $1: 5000$ dilution; Abcam, Cambridge, UK) overnight at $4^{\circ} \mathrm{C}$. After washing with TBST buffer for three times, an HRPconjugated secondary antibody diluted 1:5000 was used to recognize the primary antibody. Finally, the membrane was exposed and protein bands were analyzed using ImageJ software (NIH, Bethesda, MD).

2.6. $\mathrm{NAD}^{+}$Estimation. $\mathrm{NAD}^{+}$concentrations were determined using the NAD/NADH Quantification Kit (Sigma-Aldrich, Shanghai, China) in accordance with the manufacturer's instructions. Briefly, brain samples were homogenized with a NADH/NAD extraction buffer in a microcentrifuge tube before being spun for $5 \mathrm{~min}$ $\left(14,000 \mathrm{rpm}, 4^{\circ} \mathrm{C}\right)$ to obtain the NAD/NADH supernatant. To avoid being denatured by enzymes, supernatants were deproteinized by filtering through a $10 \mathrm{kDa}$ cut-off spin filter. For total NADH and NAD (NADtotal) detection, $50 \mu \mathrm{L}$ of the supernatant were transferred into a 96-well plate in duplicate; for NADH only detection, NAD was decomposed by heating to $60^{\circ} \mathrm{C}$ for $30 \mathrm{~min}$ before $50 \mu \mathrm{L}$ of the supernatant were also transferred into a plate in duplicate. After addition of $100 \mu \mathrm{L}$ Master Reaction Mix to each of the wells and incubation for $5 \mathrm{~min}$ at room temperature, $10 \mu \mathrm{L} \mathrm{NADH}$ Developer was added into each well and left to incubate for $1 \mathrm{~h}$ to $4 \mathrm{~h}$ at $25^{\circ} \mathrm{C}$. The absorbance was read at $450 \mathrm{~nm}$ using a Spectrophotometric multiwell plate reader (Multiskan GO, Thermo Fisher Scientific, Waltham, MA, US). Thereafter,
NAD concentrations were calculated in accordance with the following equation: $\mathrm{NAD}^{+}=\mathrm{NAD}^{+}$total $-\mathrm{NADH}$.

2.7. Behavioral Test. To assess the functional recovery after stroke in the four different groups, the modified Neurological Severity Score (mNSS score) and the Foot Fault test were performed at $1 \mathrm{~d}$ before and $1 \mathrm{~d}, 3 \mathrm{~d}, 7 \mathrm{~d}$, and $14 \mathrm{~d}$ after stroke induction $[25,26]$. In the Foot Fault test, each mouse was placed on a homemade wire grid with a camera beneath it to record the foot faults. Mice were allowed to freely walk on the wire grid for $5 \mathrm{~min}$. The movie was analyzed with a computer. A foot fault was confirmed if the paw went through the grid hole failing to provide support for the mice. Foot faults were calculated with the following equation: foot faults $\%=\{$ (number of foot faults $) /($ total footsteps $)\} \times 100$. The mNSS score includes a composite of motor, sensory, beam balance, reflex absence, and abnormal movement tests as previously reported $[25,26]$. The observers analyzing the video and recording the results were blind to the experimental conditions.

2.8. Statistics. Data were analyzed with SPSS v.19.0 software (SPSS Inc., Chicago, IL, USA) and are shown as the mean \pm standard deviation (SD). One-way analysis of variance (ANOVA) and Bonferroni posttests were used to evaluate differences among the groups; $p<0.05$ was considered statistically significant.

\section{Results}

3.1. NAM Administration Led to Reduced Infarct Size, Increased Fractional Anisotropy Value, and Fiber Counts after Stroke. In vivo T2WI and DTI were carried out at $7 \mathrm{~d}$ and $14 \mathrm{~d}$ in the four groups. Reduction of infarct sizes was observed at $7 \mathrm{~d}$ of continuous administration of NAM as shown on T2-weighted images (Figures $1(\mathrm{a})$ and $1(\mathrm{~d})$ ). When comparing fractional anisotropy (FA) values in the IC (illustrated in the color map in Figure 1(b)) between the four groups at $7 \mathrm{~d}$ after stroke, no significant differences were found (Figure 1(e)). However, at $14 \mathrm{~d}$, the increase in FA values in the NAM group was significantly higher compared to that in the other three groups (Figure 1(f)). In addition, the fiber counts in the IC were also significantly higher in the NAM group compared to those in the other three groups (Figures $1(\mathrm{c})$ and $1(\mathrm{~g})$ ). These results suggested a better recovery of myelination with NAM administration after stroke.

Moreover, analysis of immunofluorescence staining showed that at $7 \mathrm{~d}$ after NAM administration, O4 staining, a marker of pro-oligodendrocytes, was higher in the NAM group than that in the other three groups in the subventricular zone (Figures 2(a) and 2(b) and Supplementary Figure 1 available online at https://doi.org/10.1155/2017/ 7019803), indicating that more mature oligodendrocytes were generated and thus supporting the evidence found with MRI. Western blot analysis and immunofluorescence staining for MBP, a marker for mature oligodendrocytes, showed that MBP expression was higher in the NAM treated group than that in the other three groups in the peri-infarct area 


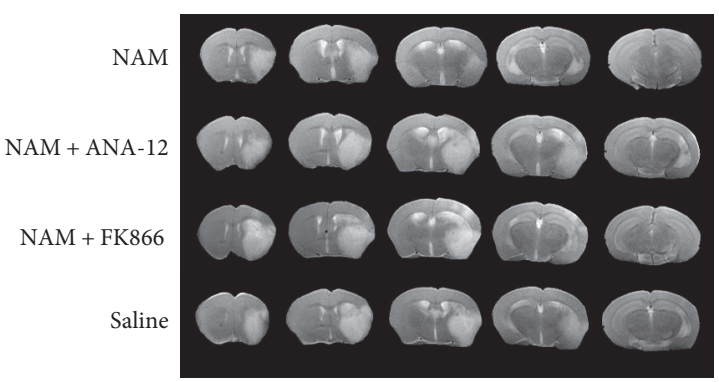

(a)

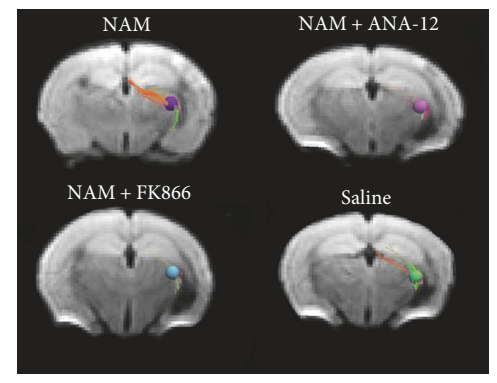

(c)

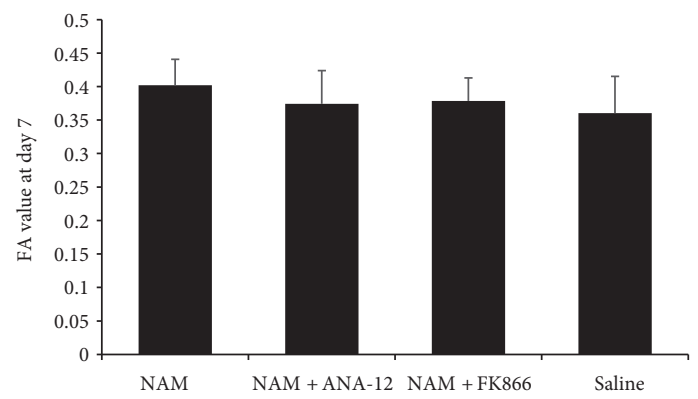

(e)

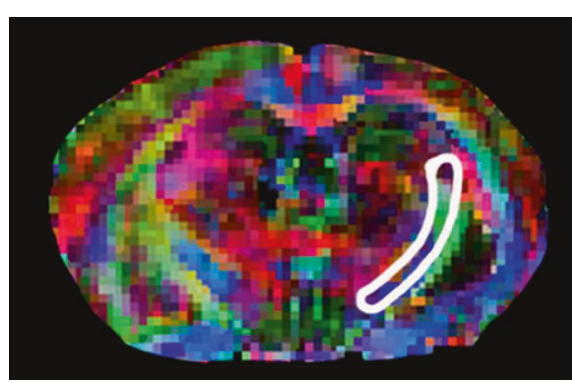

(b)

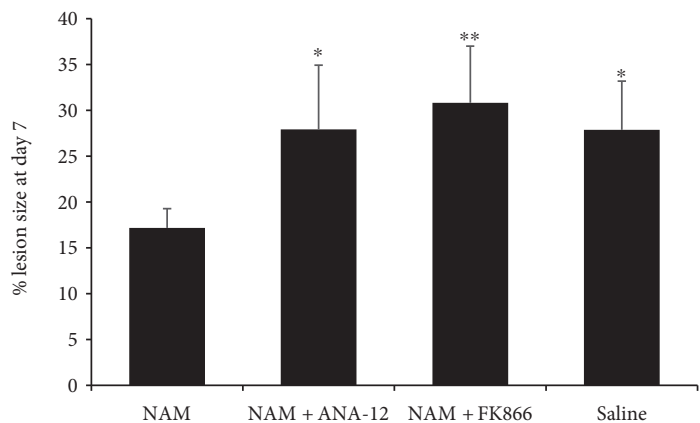

(d)

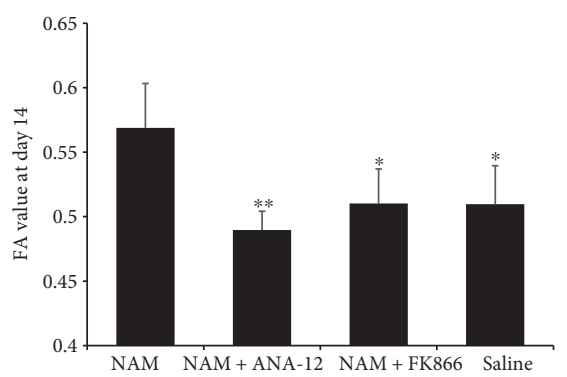

(f)

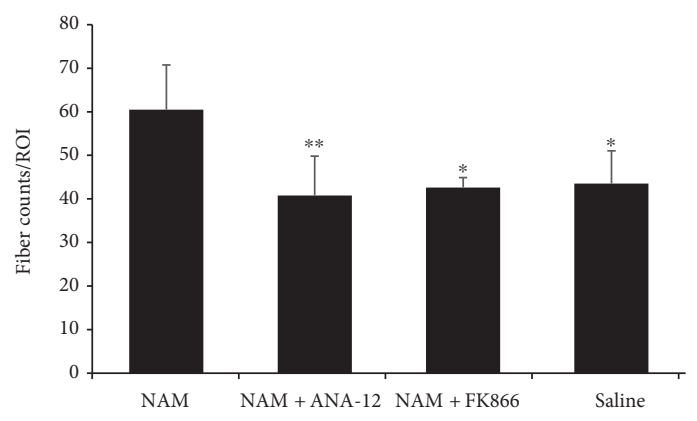

(g)

FIGURE 1: Increased remyelination after NAM administration was observed in vivo with MRI. (a) T2-weighted images showing the reduction of infarct size as observed at $7 \mathrm{~d}$ after NAM injection. (b) Representative FA color map of a brain section. The area surrounded by white line was the site of internal capsule selected for FA value determining. (c) Representative photographs of fiber tracking in the region of interest (ROI) of IC from the four groups. ROI radius: $0.45 \mathrm{~mm}$. The directions of fiber tracks are color-coded with red for left-right, blue for superior-inferior, and green for anterior-posterior. (d) Quantification of the infarct size in the four groups. $n=6$. (e) FA values measured in the IC at $7 \mathrm{~d}$ after stroke induction. $n=7$ for the NAM group; $n=6$ for the FK866 group; $n=5$ for the NAM+ANA-12 and saline groups. (f) FA values measured in the IC at $14 \mathrm{~d}$ after stroke induction. $n=7$ for the NAM group; $n=6$ for the FK866 group; $n=5$ for the NAM+ANA-12 and saline groups. (g) Quantitative data of fiber counts performed in the ICs of the four groups. $n=7$ for the NAM group; $n=6$ for the FK866 group; $n=5$ for the NAM+ANA-12 and saline groups. Data showed as mean \pm SD in all histograms. ${ }^{*} p<0.05$, ${ }^{* *} p<0.01$ versus NAM. 


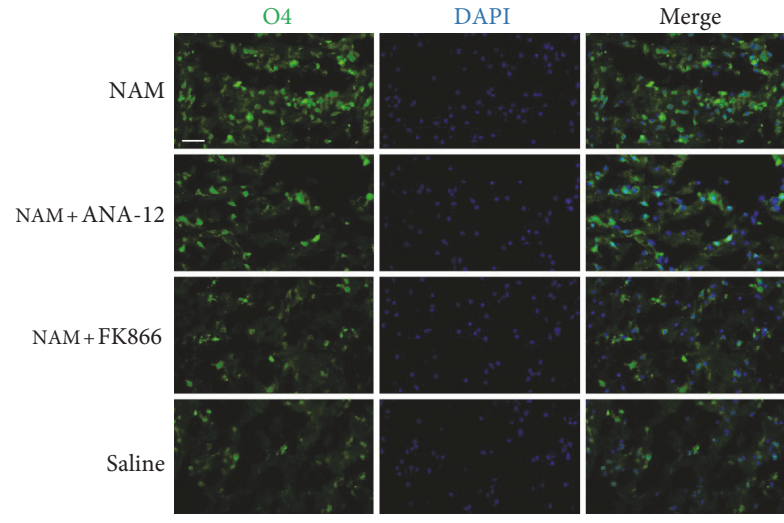

(a)

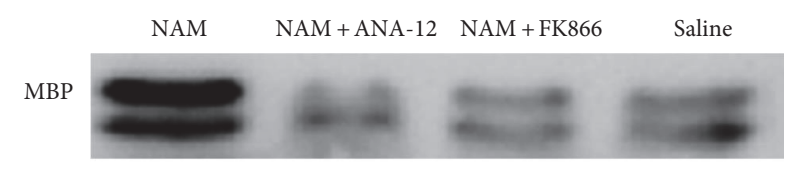

$\beta$-Actin

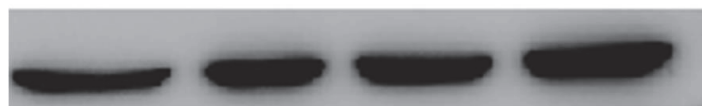

(c)

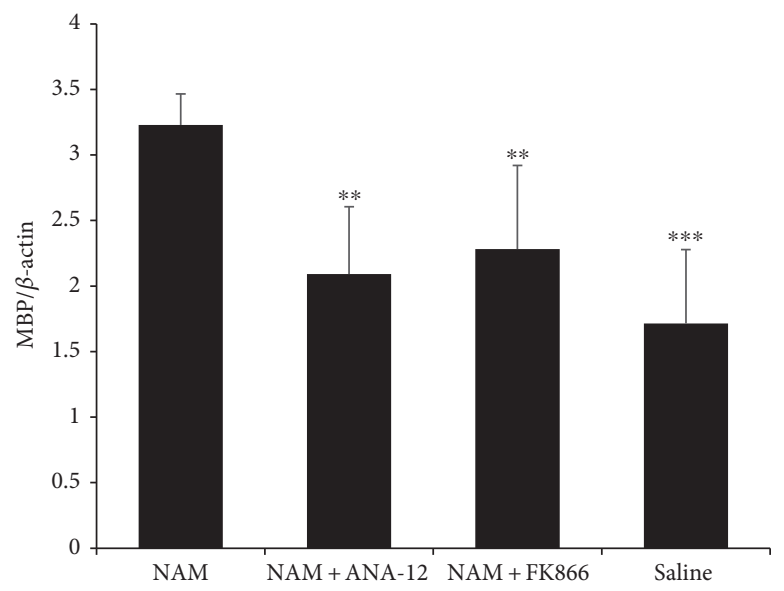

(d)

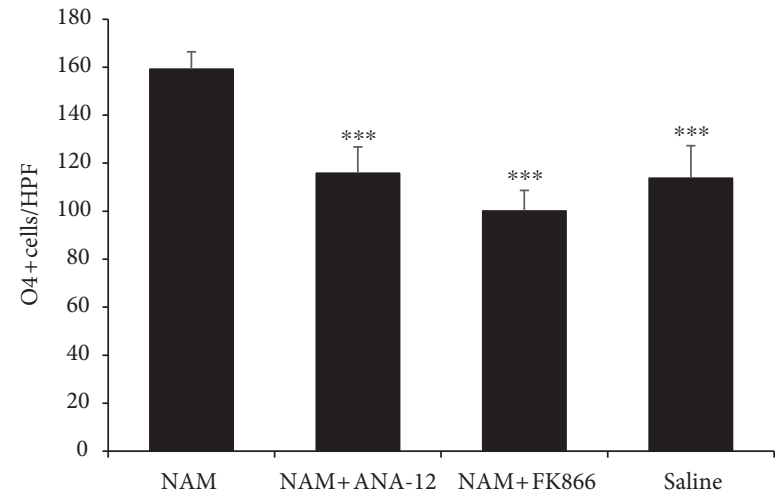

(b)

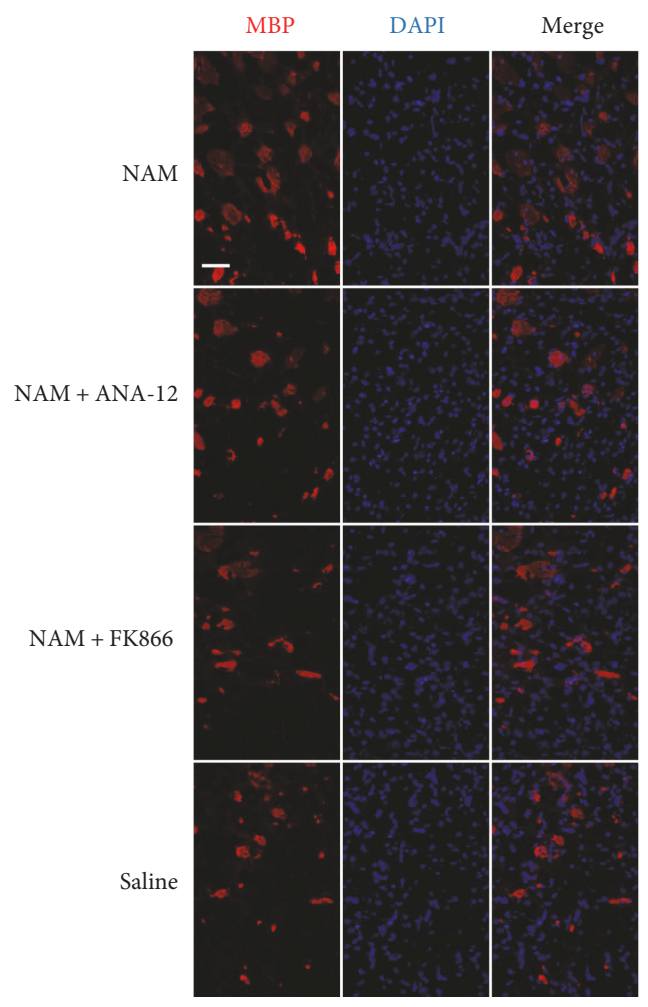

(e)

Figure 2: Continuous NAM administration enhanced mature oligodendrocytes generation and remyelination after stroke. Immunofluorescence images (a) and quantitative data $(n=4)$ (b) showing an increase in $\mathrm{O} 4$ expression in the NAM group at $7 \mathrm{~d}$ after stroke induction. Western blot analysis (c), quantitative data $(n=8)(\mathrm{d})$, and immunofluorescence images (e) showing that MBP expression was higher in the NAM group compared to the other three groups at $14 \mathrm{~d}$ after stroke induction. Data showed as mean \pm SD in all histograms. ${ }^{* *} p<0.01,{ }^{* * *} p<0.001$ versus NAM. HPF: high power field. Scale bar: $40 \mu \mathrm{m}$.

at $14 \mathrm{~d}$ (Figures 2(c), 2(d), and 2(e) and Supplementary Figure 2), further supporting our MRI results.

3.2. Nicotinamide Administration Promoted the Expression of $B D N F$ via Increased $N A D^{+}$Levels. To verify whether continuous NAM administration could increase $\mathrm{NAD}^{+}$levels in the peri-infarct area of mouse brain long after the stroke, we evaluated the levels of $\mathrm{NAD}^{+}$at $7 \mathrm{~d}$ after stroke induction. As expected, $\mathrm{NAD}^{+}$levels were significantly higher in the NAM-treated group than those in the saline-treated group.
FK866, an inhibitor of NAMPT, prevented the conversion of NAM into $\mathrm{NAD}^{+}$, resulting in a lower $\mathrm{NAD}^{+}$level in the $\mathrm{NAM}+\mathrm{FK} 866$ group, although mice in this group received continuous NAM administration. In contrast, ANA-12, a TrkB-selective antagonist, had no effect on the NAMinduced increased level of $\mathrm{NAD}^{+}$(Figure 3).

To determine whether NAM-induced $\mathrm{NAD}^{+}$elevation affects BDNF expression after stroke, we assessed expression of BDNF in the peri-infarct area of the brain. Our results showed that the expression of BDNF was significantly higher 


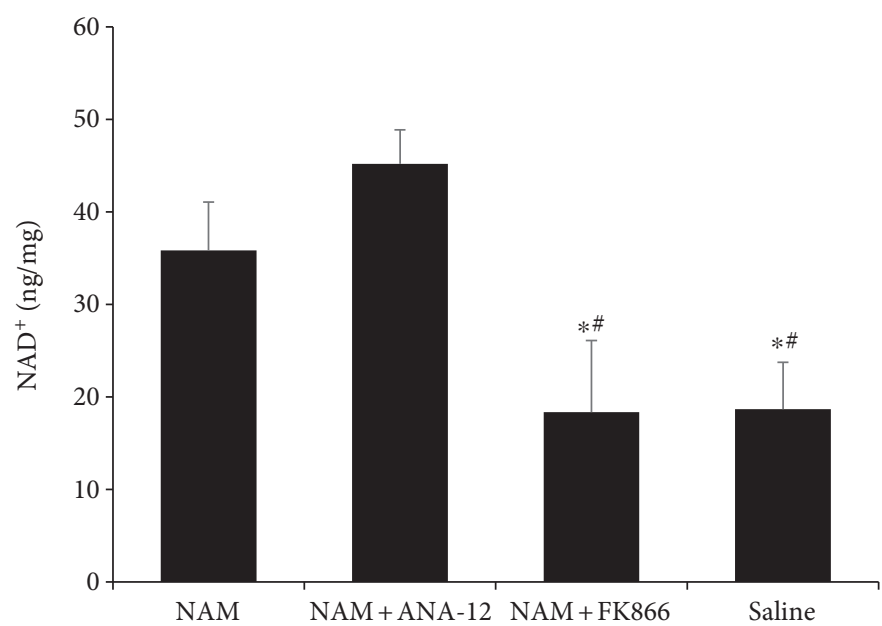

FIgURE 3: Continuous treatment with NAM induced an increased in NAD ${ }^{+}$level; FK866 treatment blocked the conversion of NAM into $\mathrm{NAD}^{+}$via inhibition of NAMPT enzyme activity. Data showed as mean \pm SD in histograms. $n=3 ;{ }^{*} p<0.05$ versus NAM; ${ }^{*} p<0.05$ versus NAM+ANA-12.

in NAM-treated and NAM+ANA-12-treated groups at $7 \mathrm{~d}$ after stroke than BDNF expression level in the NAM+ FK866-treated and saline-treated groups (Figures 4(a) and $4(\mathrm{~b})$ ), thereby showing a similar trend in $\mathrm{NAD}^{+}$levels detected in the same groups at the same time point. Higher levels of BDNF were observed in the NAM-treated and NAM+ANA-12-treated groups than in the NAM+FK866treated and saline-treated groups at $14 \mathrm{~d}$ after induction of the experimental stroke (Figures 4(d) and 4(e)). These findings were confirmed by immunofluorescence staining (Figures 4(c) and 4(f)).

3.3. Elevated BDNF Interacts with TrkB to Promote TrkB Phosphorylation after Stroke. To elucidate the interaction between BDNF and its receptor TrkB, we used a selective TrkB antagonist to block BDNF binding. As shown in Figure 5, we found no difference in $\operatorname{TrkB}$ expression among the four groups at $14 \mathrm{~d}$. However, phosphorylated TrkB (P-TrkB) expression was significantly higher in the NAM group than in the other three groups. Treatment with ANA-12 blocked NAM-induced phosphorylation of TrkB in the brain.

3.4. Nicotinamide Enhanced Functional Recovery after Stroke. To determine whether NAM continuous administration had an effect on functional recovery after stroke, mNSS scores, and Foot Fault test were performed before stroke induction and $1 \mathrm{~d}, 3 \mathrm{~d}, 7 \mathrm{~d}$, and $14 \mathrm{~d}$ after stroke. No significant differences in these test results were observed $1 \mathrm{~d}$ and $3 \mathrm{~d}$ after stroke induction. However, at $7 \mathrm{~d}$ and $14 \mathrm{~d}$ after stroke, mice treated with NAM showed a statistically significant reduction of neurological functional deficits compared to those of the other three groups (Figure 6). Mice in the NAM group showed increased motor, sensory, and cognitive functions. Both FK866 and ANA-12 blocked NAM's effect on functional recovery.

\section{Discussion}

Considering the important role of myelin in the brain, it is necessary to emphasize the importance of remyelination poststroke recovery. Assessment of the effects of NAM on ischemic stroke recovery has been carried out extensively. However, since most of the studies have focused on the acute phase of ischemic stroke mostly, they emphasized the protective effects of NAM on cells' death (such as neurons and vascular cells) during ischemic stroke [19, 27, 28]. In our experiments, NAM exerted a positive role in remyelination after stroke. During NAM treatment, a significant increase of $\mathrm{O} 4$ expression was observed in the subventricular zone $7 \mathrm{~d}$ after experimental stroke induction, indicating that more mature oligodendrocytes were generated, thereby aiding the remyelination process. In addition, $14 \mathrm{~d}$ after NAM treatment, more cells in the peri-infarct area showed expression of MBP, a specific maker of mature oligodendrocytes. The results suggested that treatment with NAM induced more pro-oligodendrocytes to transform into mature ones resulting in a better recovery of myelination after stroke. MRI has been extensively used to evaluate therapy efficiency and predict the recovery after ischemic stroke. MRI monitors noninvasively biological processes at a high resolution and in real time. Furthermore, by using MRI, we obtained a series of consecutive images in the same subject, thereby obtaining more detailed information by processing the statistic power of longitudinal studies. MRI also enables repetitive imaging in the same subject. Within the organized glial bundles surrounding the tightly packed and coherently aligned axons, the movement of water molecules perpendicular to the orientation of axons were hindered to a greater extent compared to the movements parallel to the axon orientation, resulting in anisotropic diffusion [29]. It has been reported that myelinated axons could strongly restrict diffusion of water in the white matter [30]. 


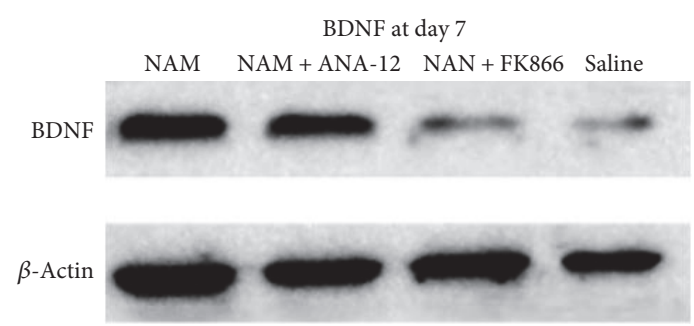

(a)

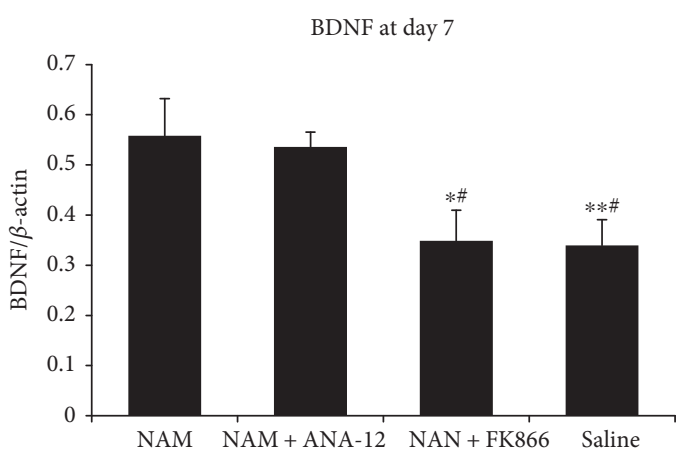

(b)

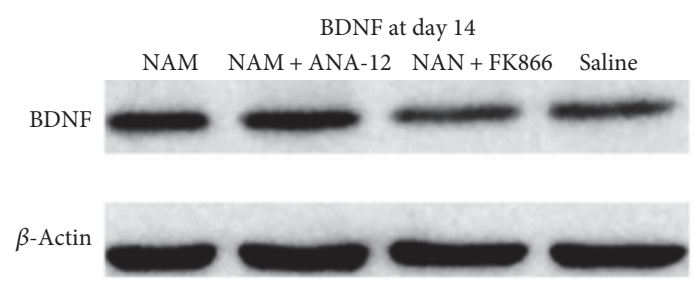

(d)

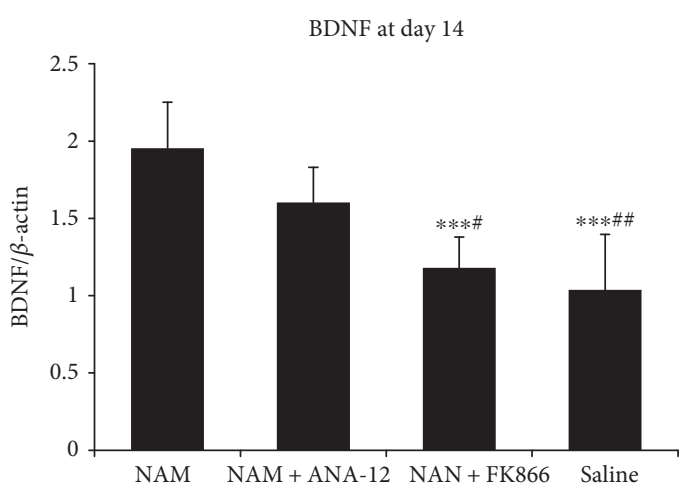

(e)

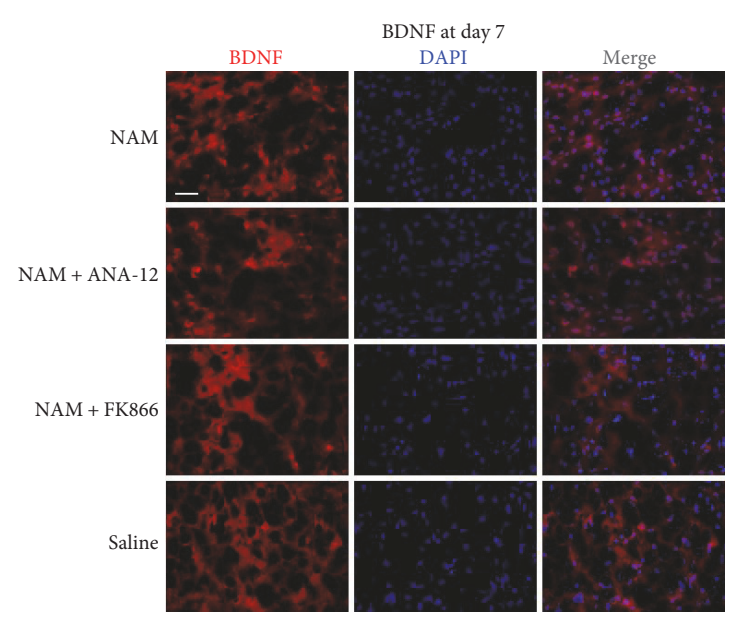

(c)

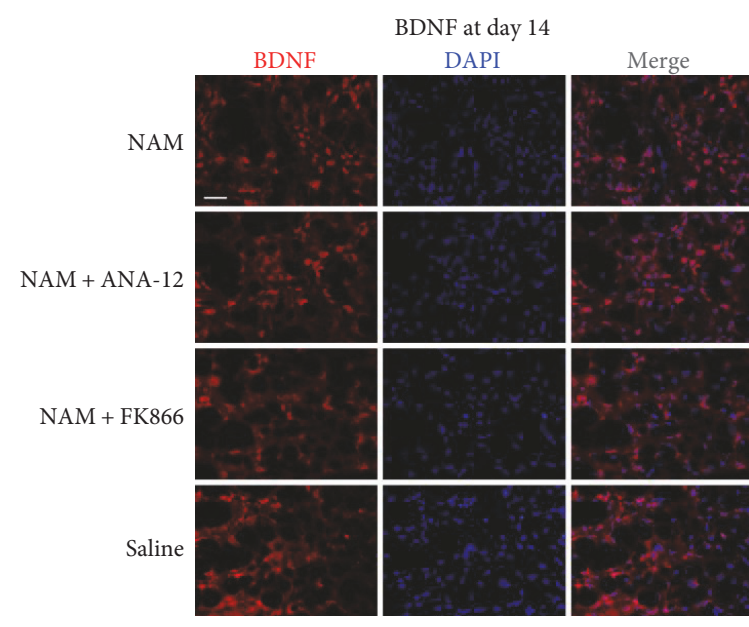

(f)

Figure 4: Western blot analysis (a), quantitative data $(n=3)(\mathrm{b})$, and immunofluorescence images (c) showing BDNF expression $7 \mathrm{~d}$ after stroke induction. Western blot analysis $(\mathrm{d})$, quantitative data $(n=4)(\mathrm{e})$, and immunofluorescence images $(\mathrm{f})$ showing BDNF expression $14 \mathrm{~d}$ after stroke induction. Data showed as mean $\pm \mathrm{SD}$ in all histograms. ${ }^{*} p<0.05,{ }^{* *} p<0.01,{ }^{* * *} p<0.001$ versus NAM; ${ }^{*} p<0.05$, $\# p<0.01$ versus NAM+ANA-12. Scale bar: $40 \mu \mathrm{m}$.

Therefore, we sought to evaluate the recovery of myelination after stroke by analyzing fractional anisotropy (FA) of water diffusion obtained from DTI in vivo with MRI scanning. Moreover, fiber counts were also assessed after reconstruction of DTI to assess the recovery of myelination.
We carried out T2WI and DTI with a 7 T small animal magnetic resonance system $7 \mathrm{~d}$ and $14 \mathrm{~d}$ after experimental stroke induction. A significant decrease of the infarct size was observed in the NAM group; however, no significant differences in the FA values obtained from DTI were observed in 


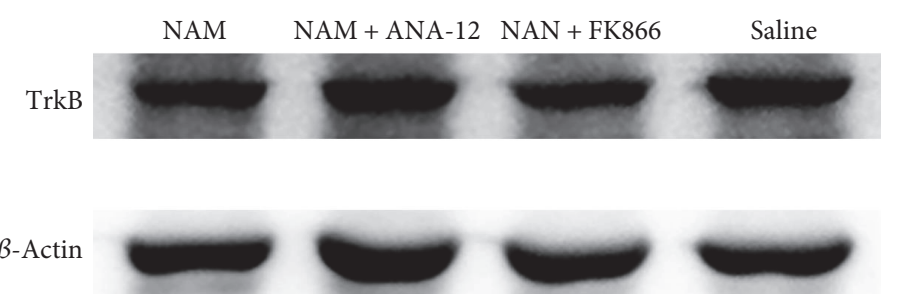

(a)

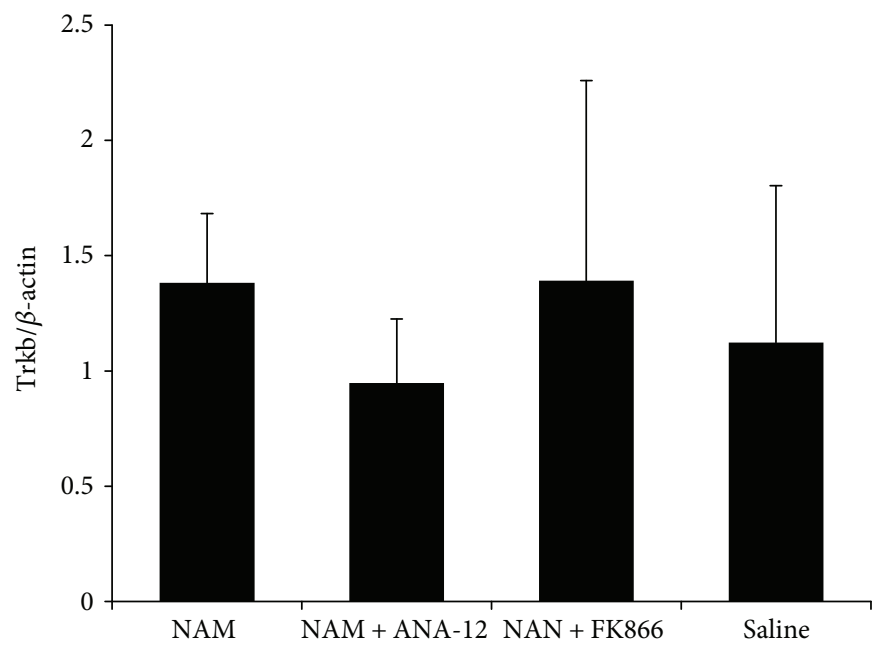

(b)

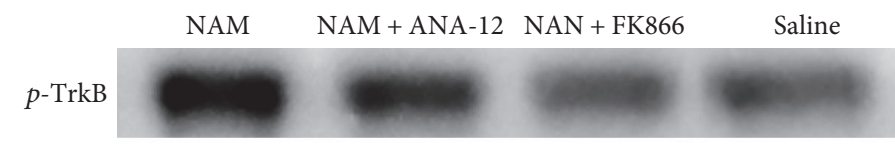

$\beta$-Actin

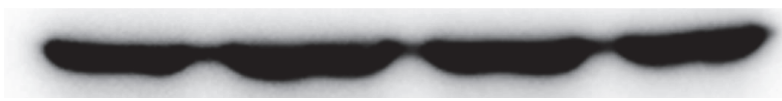

(c)

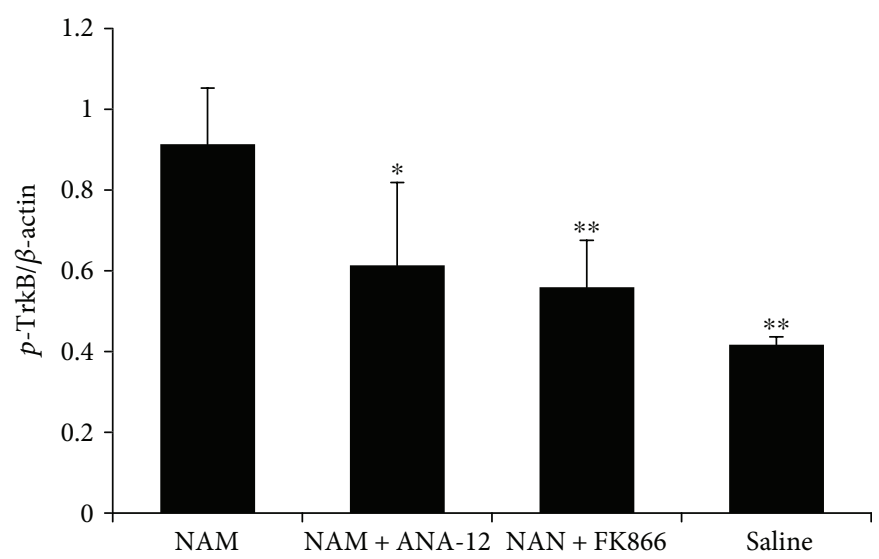

(d)

FIGURE 5: BDNF interacts with TrkB to promote remyelination after stroke. Western blot analysis (a) and quantification data (b) showing no differences between TrkB expressions in the four groups. Western blot analysis (c) and quantification data (d) show that the phosphorylated TrkB (P-TrkB) level was higher in the NAM group compared to the other groups. Data showed as mean \pm SD in all histograms. $n=4$; ${ }^{*} p<0.05,{ }^{* *} p<0.01$ versus NAM. 


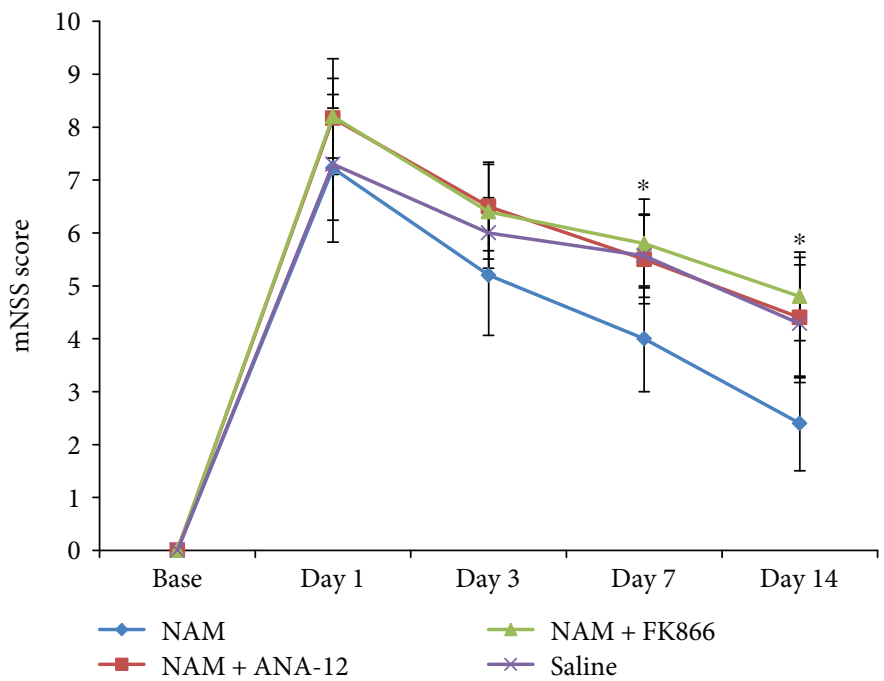

(a)

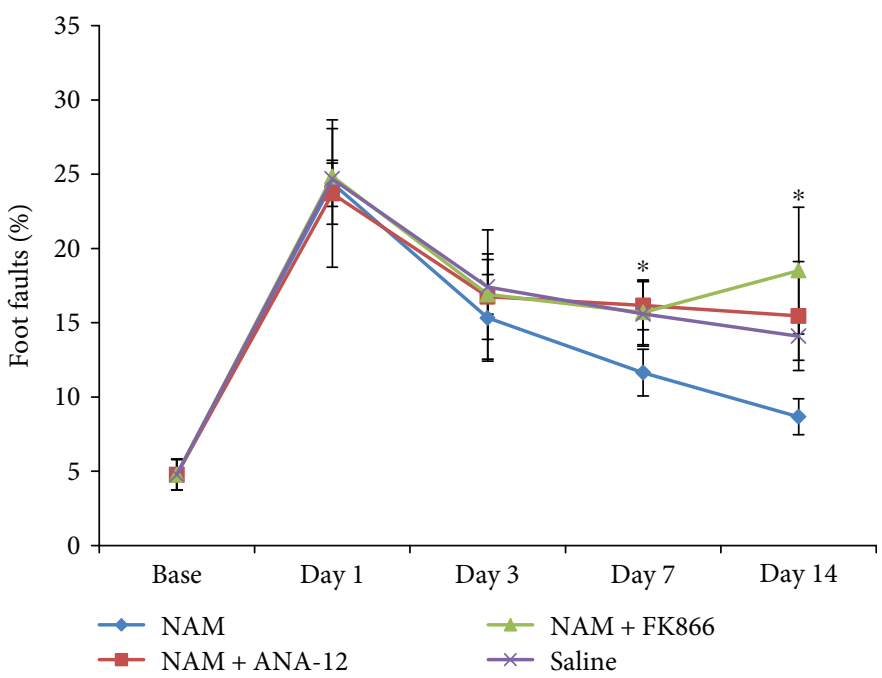

(b)

FIGURE 6: Effect of nicotinamide on functional recovery after stroke. NAM treatment resulted in time-dependent functional recovery after stroke. (a) The modified neurological severity score (mNSS) ( $n=8$ for the NAM and saline groups; $n=5$ for the NAM+FK866 and NAM+ ANA-12 groups) and (b) Foot Fault tests were performed before stroke induction and $1 \mathrm{~d}, 3 \mathrm{~d}, 7 \mathrm{~d}$, and $14 \mathrm{~d}$ after stroke induction $(n=5)$. Data showed as mean \pm SD in all line charts. ${ }^{*} p<0.05$ versus NAM.

the IC $7 \mathrm{~d}$ after the stroke. A significant increase of FA values was observed after $14 \mathrm{~d}$ in the NAM group, suggesting that myelinated axons increased after NAM administration, reflecting the occurrence of the remyelination process after the stroke. DTI analysis showed an increase in fiber counts in the NAM group. A similar trend was observed for $\mathrm{O} 4$ and MBP expressions detected with Western blot analysis and immunofluorescence staining. To our knowledge, this is the first study showing that NAM administration could promote remyelination after stroke. Owing to the important role of myelin in maintaining correct axonal conduction velocity across the brain, functional recovery assessment is useful to evaluate the extent of myelination recovery. Previous studies have confirmed that mNSS and Foot Fault tests are powerful to evaluate the functional recovery after stroke $[31,32]$. In this study, NAM treatment induced a significant improvement in functional recovery $7 \mathrm{~d}$ and $14 \mathrm{~d}$ after stroke compared to the other study groups.

Brain-derived neurotrophic factor (BDNF) is one of the most important trophic factors in the brain. BDNF has been shown to modulate neuronal circuits, promote neurogenesis, increase synaptic plasticity and axon growth, and enhance angiogenesis [33]. BDNF binds to two different receptors: the tropomyosin-related kinase receptor B (TrkB) and the p75 neurotrophin receptor (p75 NTR) [34, 35]. It has been reported that interactions between BDNF and TrkB potentiate CNS myelination during early postnatal development. BDNF promotes oligodendroglia maturation and myelination during development [36]. In vitro and BDNF knockout studies also emphasize the important effects of BDNF on myelination $[37,38]$. To investigate whether BDNF played an important role in the remyelination process after NAM 
treatment in ischemic stroke, we detected the expression of BDNF in the peri-infarct area $7 \mathrm{~d}$ and $14 \mathrm{~d}$ after stroke induction. Results suggested that NAM enhanced the expression of BDNF after stroke, which correlates with enhanced recovery of myelination. To determine whether BDNF/TrkB pathway plays a role in the remyelination process during stroke recovery, we tested the effect of ANA-12, a selective TrkB antagonist. Our results showed that ANA-12 effectively blocked the phosphorylation of TrkB, and accordingly no better remyelination was observed in the NAM+ANA-12treated group, despite higher BDNF expression being observed in the NAM-treated and NAM+ANA-12-treated groups. This result confirmed the important role played by BDNF in remyelination after stroke and suggested that NAM administration promotes remyelination via the BDNF/ TrkB pathway.

Although our results demonstrated that BDNF/TrkB pathway plays an important role in remyelination during NAM treatment after stroke, it is unknown how NAM enhanced BDNF expression after stroke. NAM is converted into $\mathrm{NAD}^{+}$by NAMPT in vivo via the salvage pathway, the main synthesis pathway of $\mathrm{NAD}^{+}$in mammalian cells [39]. $\mathrm{NAD}^{+}$is associated with aging, metabolism, and neurodegeneration [40]. Lower $\mathrm{NAD}^{+}$levels have been reported to reduce activity-dependent expression of BDNF owing to its effect on the increased methylation of BDNF promoter [41]. In vitro studies showed that administration of NAM could prevent $\mathrm{NAD}^{+}$depletion to protect neurons against damage from excitotoxicity, and this effect was also confirmed in vivo in an acute poststroke phase [42, 43]. However, no previous studies have investigated whether long-term NAM treatment could preserve $\mathrm{NAD}^{+}$at a higher level after stroke. In addition, we also wanted to determine whether increased BDNF expression was due to elevated $\mathrm{NAD}^{+}$after NAM administration. Our results showed that $\mathrm{NAD}^{+}$levels were higher in the NAM-treated group $7 \mathrm{~d}$ after stroke induction. When we blocked the conversion of NAM into $\mathrm{NAD}^{+}$via NAMPT inhibition by a selective (FK866), the elevated $\mathrm{NAD}^{+}$level caused by NAM administration was also abolished. This resulted in decreased BDNF expression in the NAM+ FK866-treated group. Therefore, our results suggest that NAM administration enhanced BDNF expression via increased $\mathrm{NAD}^{+}$level after stroke induction. Increased BDNF levels facilitated enhanced recovery of myelination after stroke via activation of $\operatorname{TrkB}$ receptor. The effect of NAM on remyelination was blocked by either inhibiting the activity of NAMPT to prevent NAM conversion into $\mathrm{NAD}^{+}$or blocking BDNF binding to TrkB after NAM treatment. Lacking of the two other control groups which were ANA-12 or FK866 treatment alone could be one of the limitations in this study. With them, specificity of the two inhibitors (ANA-12 and FK866) on NAM actions could have exhibited better.

\section{Conclusions}

We showed that NAM administration could promote remyelination after stroke via a $\mathrm{NAD}^{+} / \mathrm{BDNF} / \operatorname{TrkB}$ pathway. Our studies supplied a new restorative therapeutic strategy for the treatment of ischemic stroke.

\section{Conflicts of Interest}

Study design was approved by the appropriate ethics review board. All the authors have approved the manuscript. There are no conflicts of interest to declare.

\section{Authors' Contributions}

Congxiao Wang conducted all the experiments, integrated the data, edited the figures, and wrote the manuscript. Yi Zhang, Jie Ding, Zhen Zhao, Cheng Qian, and Ying Luan provided essential assistance. Gao-Jun Teng directed this study, designed the research, and gave key advices.

\section{Acknowledgments}

This study was supported by the National Key Basic Research Program of China (nos. 2013CB733800 and 2013CB733803), the National Natural Science Foundation of Jiangsu Province (no. BK20130057), the National Science Foundation for Youths of China (no. 81301870), and the Fundamental Research Funds for the Central Universities (no. 2242015k30002).

\section{References}

[1] E. H. Lo, T. Dalkara, and M. A. Moskowitz, "Mechanisms, challenges and opportunities in stroke," Nature Reviews Neuroscience, vol. 4, no. 5, pp. 399-415, 2003.

[2] The National Institute of Neurological Disorders and Stroke rt-PA Stroke Study Group, "Tissue plasminogen activator for acute ischemic stroke," The New England Journal of Medicine, vol. 333, no. 24, pp. 1581-1588, 1995.

[3] W. Hacke, M. Kaste, E. Bluhmki et al., "Thrombolysis with alteplase 3 to 4.5 hours after acute ischemic stroke," New England Journal of Medicine, vol. 359, no. 13, pp. 1317-1329, 2008.

[4] K. R. Lees, E. Bluhmki, R. Von Kummer et al., "Time to treatment with intravenous alteplase and outcome in stroke: an updated pooled analysis of ECASS, ATLANTIS, NINDS, and EPITHET trials," The Lancet, vol. 375, no. 9727, pp. 1695-1703, 2010.

[5] C. Gumbinger, B. Reuter, C. Stock et al., "Time to treatment with recombinant tissue plasminogen activator and outcome of stroke in clinical practice: retrospective analysis of hospital quality assurance data with comparison with results from randomised clinical trials," BMJ, vol. 348, p. g3429, 2014.

[6] W. Hacke, G. Donnan, C. Fieschi et al., "Association of outcome with early stroke treatment: pooled analysis of ATLANTIS, ECASS, and NINDS rt-PA stroke trials," The Lancet, vol. 363, no. 9411, pp. 768-774, 2004.

[7] M. A. Eckert, Q. Vu, K. Xie et al., "Evidence for high translational potential of mesenchymal stromal cell therapy to improve recovery from ischemic stroke," Journal of Cerebral Blood Flow \& Metabolism, vol. 33, no. 9, pp. 1322-1334, 2013.

[8] E. G. Sozmen, J. D. Hinman, and S. T. Carmichael, "Models that matter: white matter stroke models," Neurotherapeutics: The Journal of the American Society for Experimental NeuroTherapeutics, vol. 9, no. 2, pp. 349-358, 2012.

[9] M. P. Goldberg and B. R. Ransom, "New light on white matter," Stroke, vol. 34, no. 2, pp. 330-332, 2003. 
[10] C. Matute, M. Domercq, A. Perez-Samartin, and B. R. Ransom, "Protecting white matter from stroke injury," Stroke, vol. 44, no. 4, pp. 1204-1211, 2013.

[11] M. A. Hamner, T. Moller, and B. R. Ransom, "Anaerobic function of CNS white matter declines with age," Journal of Cerebral Blood Flow \& Metabolism, vol. 31, no. 4, pp. 996-1002, 2011.

[12] A. S. Saab and K.-A. Nave, "Neuroscience: a mechanism for myelin injury," Nature, vol. 529, no. 7587, pp. 474-475, 2016.

[13] K. A. Nave, "Myelination and support of axonal integrity by glia," Nature, vol. 468, no. 7321, pp. 244-252, 2010.

[14] K. K. Bercury and W. B. Macklin, "Dynamics and mechanisms of CNS myelination," Developmental Cell, vol. 32, no. 4, pp. 447-458, 2015.

[15] N. B. Hamilton, K. Kolodziejczyk, E. Kougioumtzidou, and D. Attwell, "Proton-gated $\mathrm{Ca}^{2+}$-permeable TRP channels damage myelin in conditions mimicking ischaemia," Nature, vol. 529, no. 7587, pp. 523-527, 2016.

[16] A. A. Sauve, "NAD" and vitamin $\mathrm{B}_{3}$ : from metabolism to therapies," The Journal of Pharmacology and Experimental Therapeutics, vol. 324, no. 3, pp. 883-893, 2008.

[17] G. Rennie, A. C. Chen, H. Dhillon, J. Vardy, and D. L. Damian, "Nicotinamide and neurocognitive function," Nutritional Neuroscience, vol. 18, no. 5, pp. 193-200, 2015.

[18] T. Hathorn, A. Snyder-Keller, and A. Messer, "Nicotinamide improves motor deficits and upregulates PGC-1alpha and BDNF gene expression in a mouse model of Huntington's disease," Neurobiology of Disease, vol. 41, no. 1, pp. 43-50, 2011.

[19] K. Maiese and Z. Z. Chong, "Nicotinamide: necessary nutrient emerges as a novel cytoprotectant for the brain," Trends in Pharmacological Sciences, vol. 24, no. 5, pp. 228-232, 2003.

[20] A. C. Chen, A. J. Martin, B. Choy et al., "A phase 3 randomized trial of nicotinamide for skin-cancer chemoprevention," The New England Journal of Medicine, vol. 373, no. 17, pp. 1618-1626, 2015.

[21] E. A. M. Gale, "European nicotinamide diabetes intervention trial (ENDIT): a randomised controlled trial of intervention before the onset of type 1 diabetes," The Lancet, vol. 363, no. 9413, pp. 925-931, 2004.

[22] V. Libri, C. Yandim, S. Athanasopoulos et al., "Epigenetic and neurological effects and safety of high-dose nicotinamide in patients with Friedreich's ataxia: an exploratory, open-label, dose-escalation study," The Lancet, vol. 384, no. 9942, pp. 504-513, 2014.

[23] Y. Y. Bai, L. Wang, D. Chang et al., "Synergistic effects of transplanted endothelial progenitor cells and RWJ 67657 in diabetic ischemic stroke models," Stroke, vol. 46, no. 7, pp. 1938-1946, 2015.

[24] R. Saiki, K. Nishimura, I. Ishii et al., "Intense correlation between brain infarction and protein-conjugated acrolein," Stroke, vol. 40, no. 10, pp. 3356-3361, 2009.

[25] X. Cui, M. Chopp, A. Zacharek, Y. Cui, C. Roberts, and J. Chen, "The neurorestorative benefit of GW3965 treatment of stroke in mice," Stroke, vol. 44, no. 1, pp. 153161, 2012.

[26] J. Chen, P. R. Sanberg, Y. Li et al., "Intravenous administration of human umbilical cord blood reduces behavioral deficits after stroke in rats," Stroke, vol. 32, no. 11, pp. 2682-2688, 2001.
[27] I. A. Ayoub, E. J. Lee, C. S. Ogilvy, M. F. Beal, and K. I. Maynard, "Nicotinamide reduces infarction up to two hours after the onset of permanent focal cerebral ischemia in Wistar rats," Neuroscience Letters, vol. 259, no. 1, pp. 21-24, 1999.

[28] T. Mokudai, I. A. Ayoub, Y. Sakakibara, E.-J. Lee, C. S. Ogilvy, and K. I. Maynard, "Delayed treatment with nicotinamide (vitamin B3) improves neurological outcome and reduces infarct volume after transient focal cerebral ischemia in Wistar rats," Stroke, vol. 31, no. 7, pp. 1679-1685, 2000.

[29] G. Ding, J. Chen, M. Chopp et al., "White matter changes after stroke in type 2 diabetic rats measured by diffusion magnetic resonance imaging," Journal of Cerebral Blood Flow \& Metabolism, vol. 37, no. 1, p. 241, 2017.

[30] S. Van Cauter, J. Veraart, J. Sijbers et al., "Gliomas: diffusion kurtosis MR imaging in grading," Radiology, vol. 263, no. 2, pp. 492-501, 2012.

[31] P. C. Li, Y. Jiao, J. Ding et al., "Cystamine improves functional recovery via axon remodeling and neuroprotection after stroke in mice," CNS Neuroscience \& Therapeutics, vol. 21, no. 3, pp. 231-240, 2015.

[32] Y. Y. Bai, X. Gao, Y. C. Wang et al., "Image-guided proangiogenic therapy in diabetic stroke mouse models using a multi-modal nanoprobe," Theranostics, vol. 4, no. 8, pp. 787-797, 2014.

[33] S. Fargali, M. Sadahiro, C. Jiang et al., "Role of neurotrophins in the development and function of neural circuits that regulate energy homeostasis," Journal of Molecular Neuroscience: $M N$, vol. 48, no. 3, pp. 654-659, 2012.

[34] M. V. Chao, "Neurotrophins and their receptors: a convergence point for many signalling pathways," Nature Reviews Neuroscience, vol. 4, no. 4, pp. 299-309, 2003.

[35] E. J. Huang and L. F. Reichardt, "Neurotrophins: roles in neuronal development and function," Annual Review of Neuroscience, vol. 24, no. 1, pp. 677-736, 2001.

[36] J. Xiao, A. W. Wong, M. M. Willingham, M. van den Buuse, T. J. Kilpatrick, and S. S. Murray, "Brain-derived neurotrophic factor promotes central nervous system myelination via a direct effect upon oligodendrocytes," Neuro-Signals, vol. 18, no. 3, pp. 186-202, 2010.

[37] M. W. Vondran, P. Clinton-Luke, J. Z. Honeywell, and C. F. Dreyfus, "BDNF+/- mice exhibit deficits in oligodendrocyte lineage cells of the basal forebrain," Glia, vol. 58, no. 7, pp. 848-856, 2010.

[38] A. Cellerino, P. Carroll, H. Thoenen, and Y.-A. Barde, "Reduced size of retinal ganglion cell axons and hypomyelination in mice lacking brain-derived neurotrophic factor," Molecular and Cellular Neuroscience, vol. 9, no. 5, pp. 397-408, 1997.

[39] M. J. Son, M. Y. Son, B. Seol et al., "Nicotinamide overcomes pluripotency deficits and reprogramming barriers," Stem Cells, vol. 31, no. 6, pp. 1121-1135, 2013.

[40] E. Verdin, "NAD ${ }^{+}$in aging, metabolism, and neurodegeneration," Science, vol. 350, no. 6265, pp. 1208-1213, 2015.

[41] J. Chang, B. Zhang, H. Heath, N. Galjart, X. Wang, and J. Milbrandt, "Nicotinamide adenine dinucleotide (NAD)regulated DNA methylation alters CCCTC-binding factor (CTCF)/cohesin binding and transcription at the BDNF locus," Proceedings of the National Academy of Sciences, vol. 107, no. 50, pp. 21836-21841, 2010. 
[42] D. Liu, R. Gharavi, M. Pitta, M. Gleichmann, and M. P. Mattson, "Nicotinamide prevents $\mathrm{NAD}^{+}$depletion and protects neurons against excitotoxicity and cerebral ischemia: $\mathrm{NAD}^{+}$ consumption by SIRT1 may endanger energetically compromised neurons," Neuromolecular Medicine, vol. 11, no. 1, pp. 28-42, 2009.

[43] F. Sadanaga-Akiyoshi, H. Yao, S. I. Tanuma et al., "Nicotinamide attenuates focal ischemic brain injury in rats: with special reference to changes in nicotinamide and $\mathrm{NAD}^{+}$levels in ischemic core and penumbra," Neurochemical Research, vol. 28, no. 8, pp. 1227-1234, 2003. 

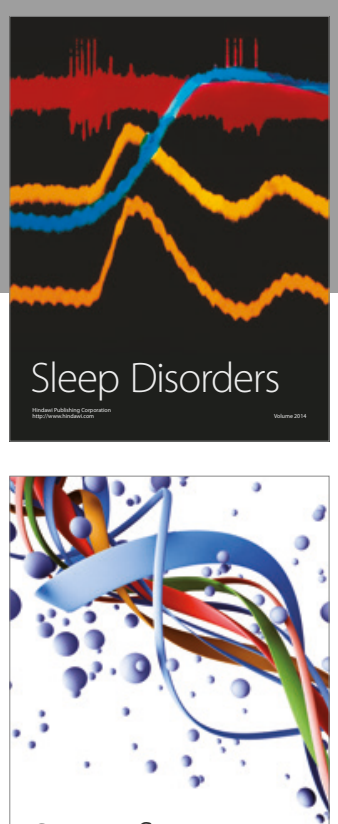

Scientifica
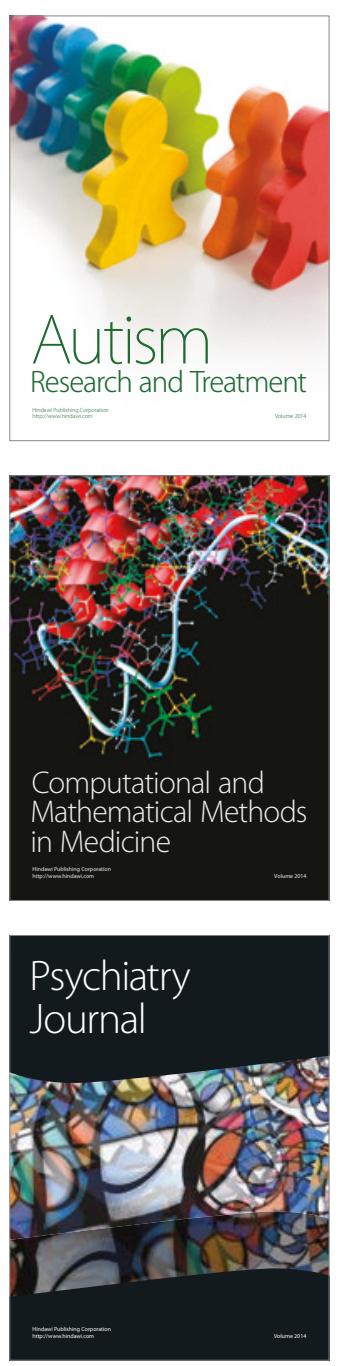
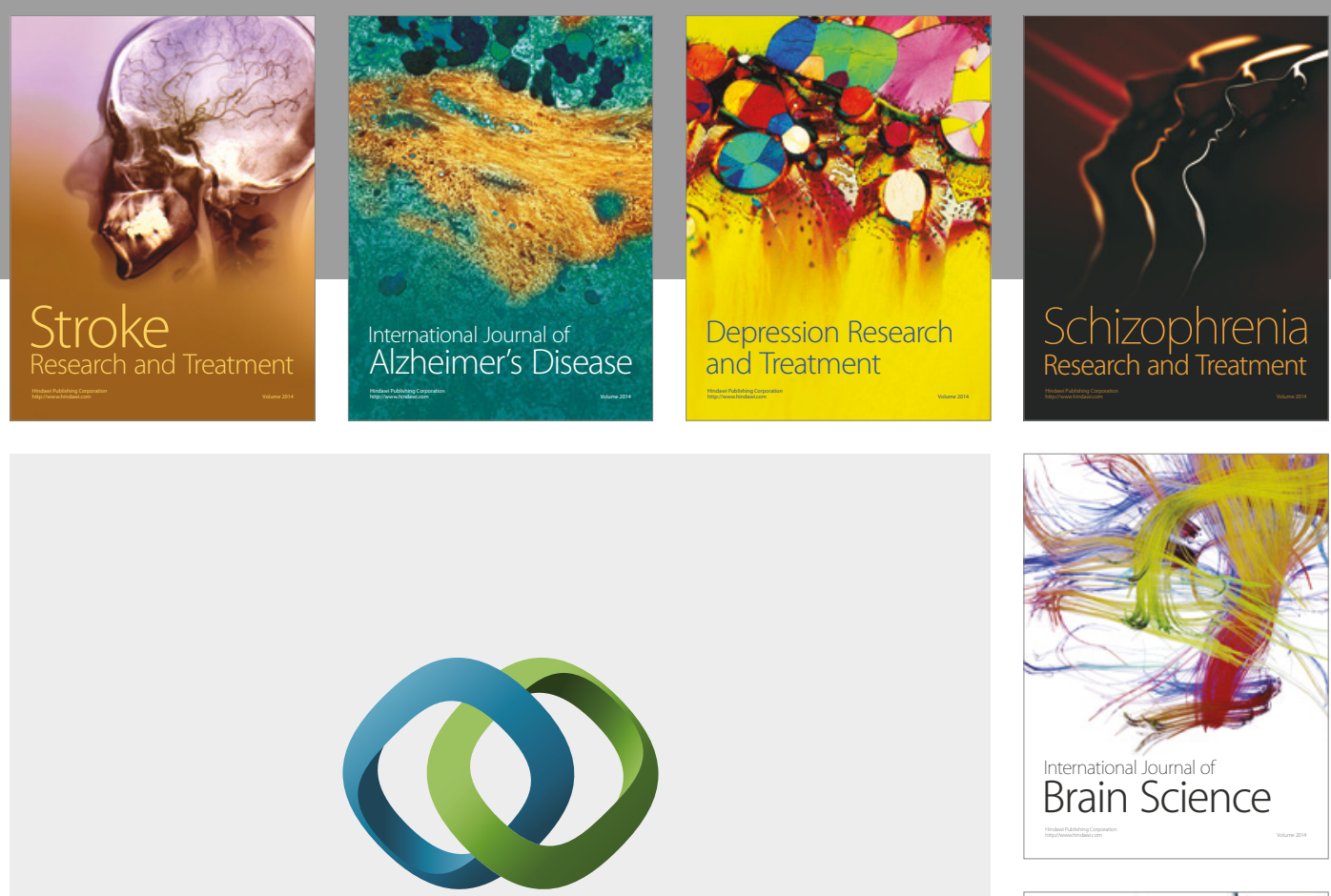

\section{Hindawi}

Submit your manuscripts at

https://www.hindawi.com
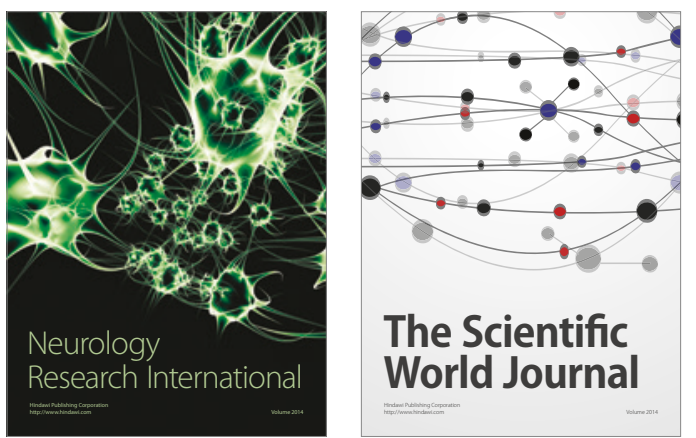

The Scientific World Journal

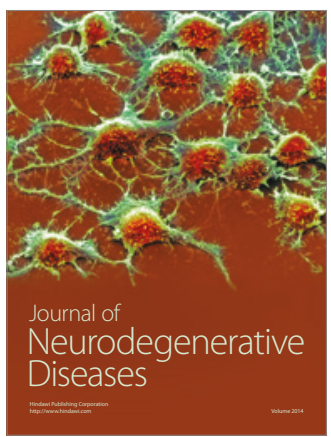

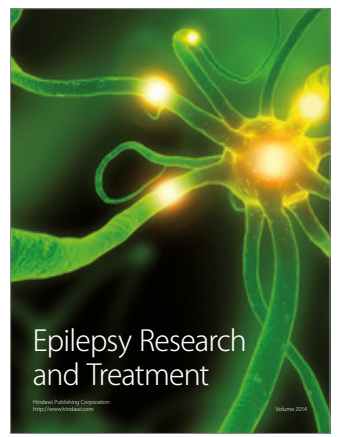

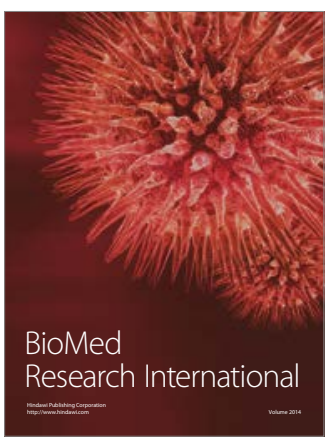

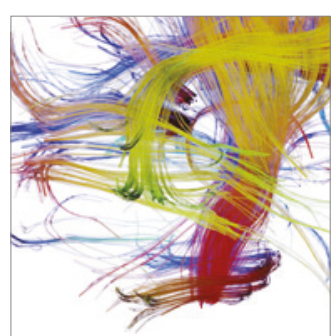

Brain Science

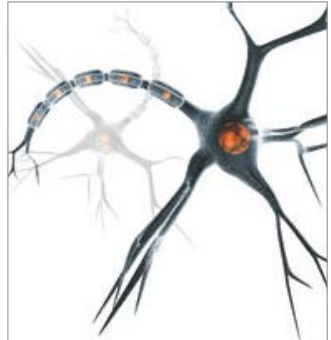

Neural Plasticity
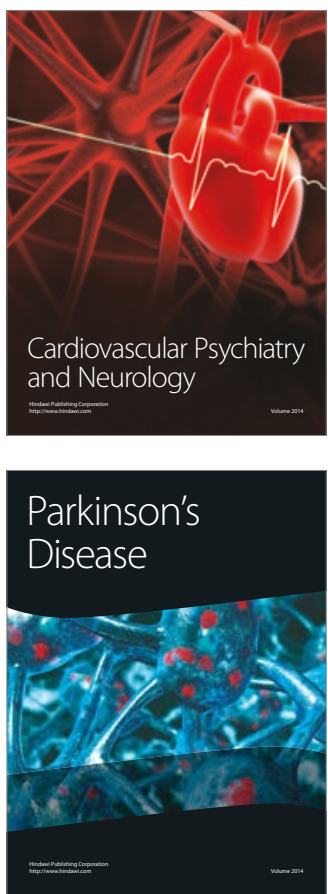